\title{
Water Ingestion ANd Pressure analysis of Automotive Vehicles USING MULTIPHASE CFD
}

Filipe Fabian Buscariolo ${ }^{1}$, Leonardo José Della Volpe ${ }^{2}$

${ }^{1}$ GM Brazil / NDF-USP

${ }^{2}$ GM Brazil

E-mail: filipe.buscariolo@gm.com, leonardo.volpe@gm.com

\begin{abstract}
Within the advances in CFD codes and HPC cluster size and processing capacities, multiphase CFD simulations became more feasible not only on research projects but also in production level. This work presents a simulation analysis of an automotive vehicle crossing certain height of water and analyzing the water ingestion by the air intake system and also the pressure load due to the water level and splashes reaching the vehicle. All the calculations were performed by Computational Fluid Dynamics, considering the car crossing $300 \mathrm{~mm}$ of water height at $20 \mathrm{kph}$ with a simulation time enough to the water reaches the whole car length. Results show the water path at the snorkel region and some part pressure load examples which can be used as an input for structural simulations to evaluate this part at this particular condition.
\end{abstract}

Keywords: CFD (computational simulation), Multiphase, Fluid-Structure Interaction (FSI).

\section{INTRODUCTION}

Within the growth of computational power, CFD simulations became a good solution to predict performance in a fluid system. This fact also made possible to perform more complex simulations in a production response time, such as multiphase fluid simulations, involving two or more fluids.

Basically, multiphase CFD simulations can be divided into two groups:

- Reactive: fluids interact chemically or physically to generate new products and properties changes. Example: combustion.

- Non-Reactive: two or more fluids are present in the computational domain, however they do not interact chemically or physically. Example: water ingestion simulation.

The present paper provides a contribution to the growing trend of using multiphase simulations in the industry. The main purpose is, by applying computational simulation, to identify water path when the vehicle is crossing a certain water height before any prototype is built, aiming to improve the vehicle's development cost and time. As a complementary study, the pressure load acting on the vehicle due to water level. 


\section{OBJECTIVES}

Based on the real condition of a car crossing certain water height, in this case $300 \mathrm{~mm}$, due to flood condition, the present work was developed aiming to reproduce this condition virtually. An example of a car crossing a flooded street is shown on Figure 1 below.

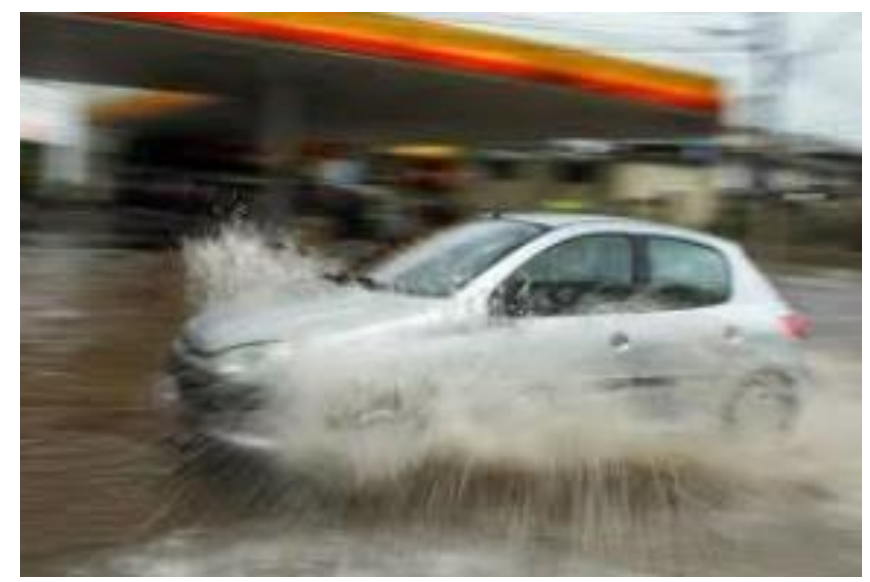

Figure 1. Car crossing flooded street.

The main objectives of this paper is to identify if water reaches engine air intake system (AIS) inlet, analyze the water path close to the vehicle and through vehicle gaps \& openings and the pressure load acting on the vehicle's due to water by using a multiphase CFD simulation.

\section{METHODOLOGY}

The methodology proposed to this work is based on correlating a physical test with proposed multiphase CFD simulation for water path. As complementary information, water pressure load will be provided by computation simulation in order to help on fixation calculation.

In order to reproduce the car trying to cross a flooded area, a test considering the car moving at $20 \mathrm{kph}$ cross a water height of $300 \mathrm{~mm}$ was performed considering a production car and the same conditions, both vehicle speed of $20 \mathrm{kph}$ and water height of $300 \mathrm{~mm}$ were applied to the multiphase CFD simulation, however in this case, the vehicle remains static and the water and the air moves toward it. A schematic representation of the tunnel inlet is shown below. 


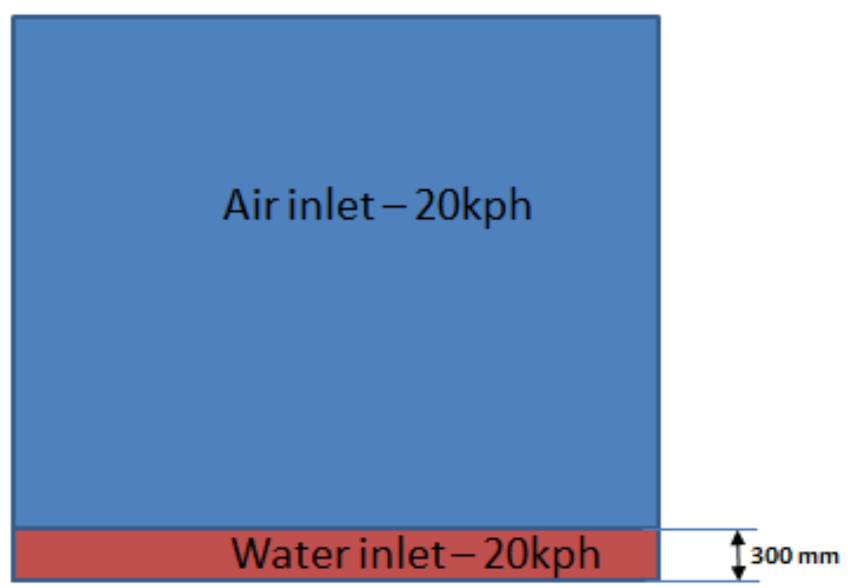

Figure 2. Wind tunnel inlet

CFD simulations were performed considering half of regular production car, in order to save computational time due to the main focus on the AIS. The simulation considered vehicle with rotating wheels, CRFM drop pressures and full underhood representation, assembled in a virtual Wind Tunnel, with dimensions similar to GM Wind Tunnel [6], once it has width similar to regular a street. Parameters considered in this work are show on table 1.

Table 1. Parameters used for CFD simulations

\begin{tabular}{|c|c|}
\hline Wind Tunnel Structure & General Motors (similar) \\
\hline Air Speed & $20 \mathrm{~km} / \mathrm{h}$ \\
\hline Outlet Pressure & Atmospheric Pressure \\
\hline Turbulence Intensity & $0.60 \%$ \\
\hline Boundary Layer Suction & Beginning of the Test \\
\hline Test Section Dimensions & $(5.4 \times 10.4 \times 10.0) \mathrm{m}$ \\
\hline Turbulence Model & $\mathrm{k}-\varepsilon$ \\
\hline
\end{tabular}

The ground simulation for this paper is moving ground with rotating wheels as mentioned before, which is the condition close to the real operating condition, according to [1] and [8] and the turbulence model considers swirl flow due to water perturbations.

\section{Model Additional Considerations}

For the model configuration of the multiphase, it was considered the Volume of Fluid (VOF) method which is a numerical technique for tracking and locating the free surface. It belongs to the class of Eulerian methods which are characterized by a mesh that is either stationary or is moving in a certain prescribed manner to accommodate the evolving shape of the interface.

VOF is an advection scheme-a numerical recipe that allows the programmer to track the shape and position of the interface, but it is not a standalone flow solving algorithm. The Navier-Stokes equations describing the motion of the flow have to be solved separately. [2] 
The turbulence model considered was the K-epsilon $(\mathrm{k}-\varepsilon)$ which is the most common model used in Computational Fluid Dynamics (CFD) to simulate turbulent conditions. It is a two equation model which gives a general description of turbulence by means of two transport equations. The first transported variable determines the energy in the turbulence and is called turbulent kinetic energy $(\mathrm{k})$. The second transported variable is the turbulent dissipation (epsilon) which determines the rate of dissipation of the turbulent kinetic energy.

The simulation was set as a transient with a fixed time step lower enough to the simulation reach convergence and represent the flow path. The total simulated time is calculated by dividing the domain length, which is $10 \mathrm{~m}$ in this case, by the speed of $20 \mathrm{kph}$ which gives a result of around 2 seconds to be simulated. In order to have stable flow, total simulated time was 4 seconds.

\section{RESULTS}

The proposed methodology and model configuration were capable to reproduce a multiphase flow of a vehicle crossing a $300 \mathrm{~mm}$ water height at constant speed of $20 \mathrm{kph}$ in a production level response time.

Results show good correlation on the water path over the car body as shown below:

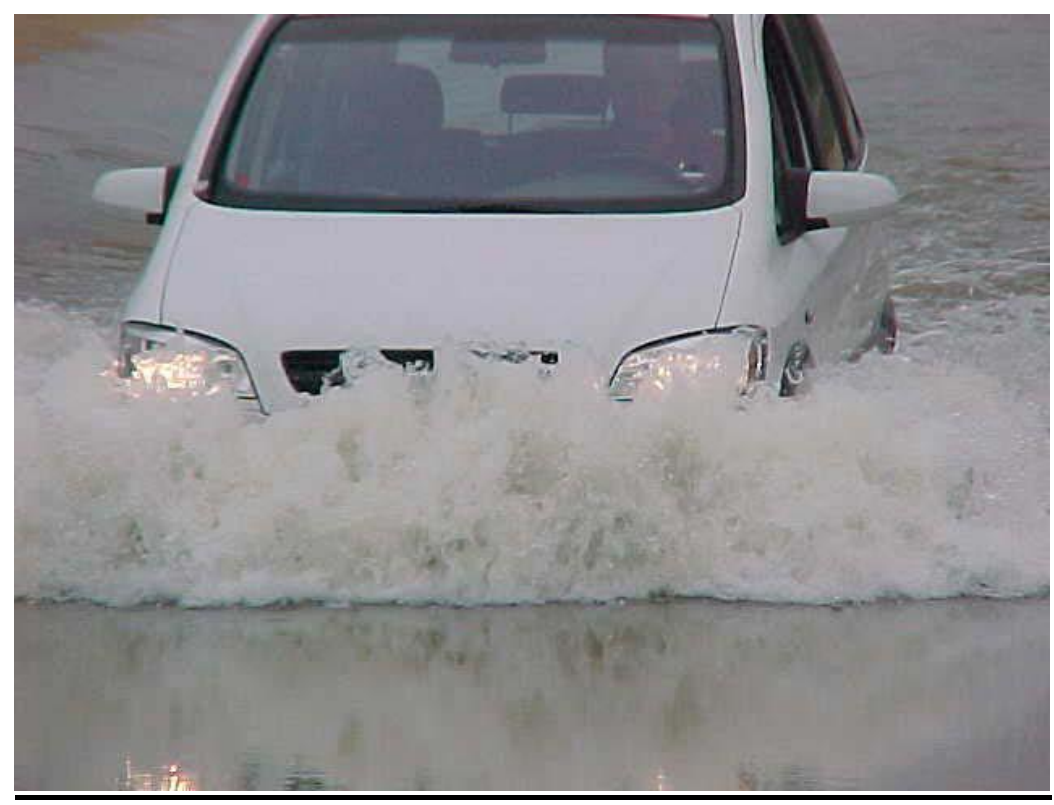

Figure 3. Water path on a production car-test 


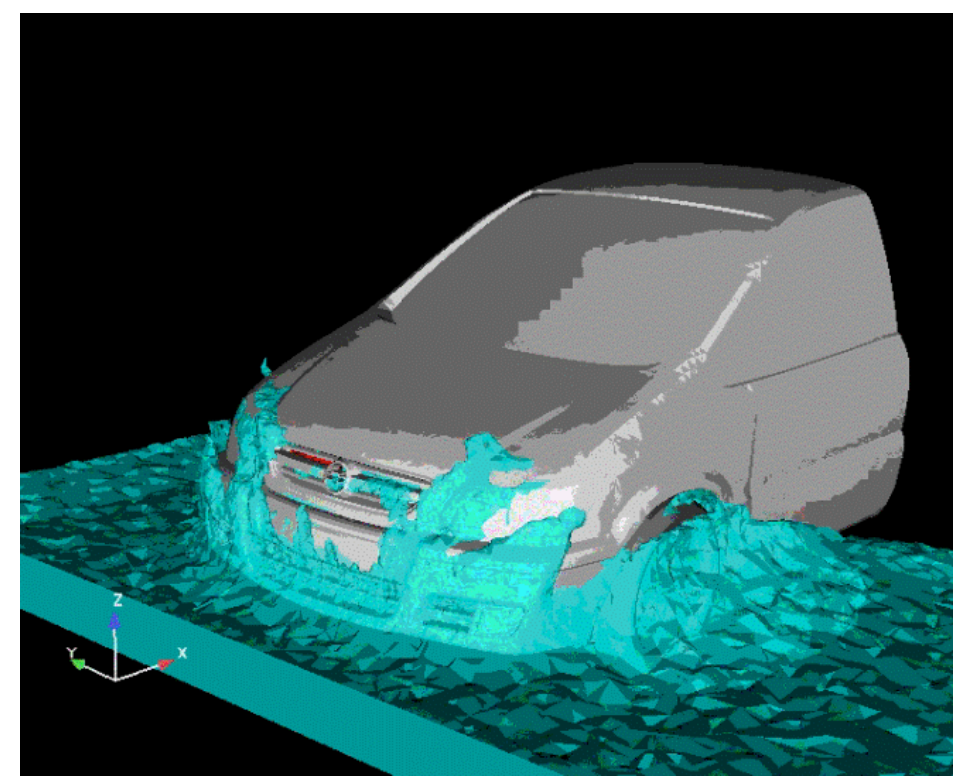

Figure 4. Water path on a production car-multiphase CFD Simulation

\section{CONCLUSION}

Automakers are always seeking for solutions to improve vehicles development cost and time. The use of computational simulations is becoming a common practice inside the industries nowadays. Considering the hardware and software improvements among the years, the level of complexity of the Computer Aided Engineering (CAE) simulations are also growing.

The use of CFD simulations are also increasing among automakers in order to improve vehicle's development time and also save cost with physical tests.

Nowadays, CFD codes have more powerful algorithms and, together with the increment of the number of processors, multiphase analysis, which are fluid simulations that consider two or more fluids in the same domain, become a nice solution and in a reliable production time.

In the present work, a methodology to perform a multiphase CFD simulation of a car crossing a water height of $300 \mathrm{~mm}$ at $20 \mathrm{kph}$, which represents a flood condition, was presented with good correlation results to the physical test.

Once the main focus of the paper was the AIS inlet, only half of the car was considered in order to speed up results and solution. Model was set considering VOF approach and Kepsilon turbulence model. Total simulated time was 4 seconds in a transient condition.

The methodology was capable to reproduce the water splash effect on the vehicle crossing it, as shown before, with a good correlation with physical test. This fact is really important once this approach can be used at early stages of the project to evaluate part performance and also save money by improving the design and performance of certain parts before any physical test. 


\section{Air Intake System Water Ingestion Analysis and Conclusions}

By analyzing the Air Intake System performance, which was the focus of this paper, computational simulation predicted water suction by the AIS and reaching the air filter as shown on Figure 5, which was taken from the virtual test at 4 seconds of the total simulation time.

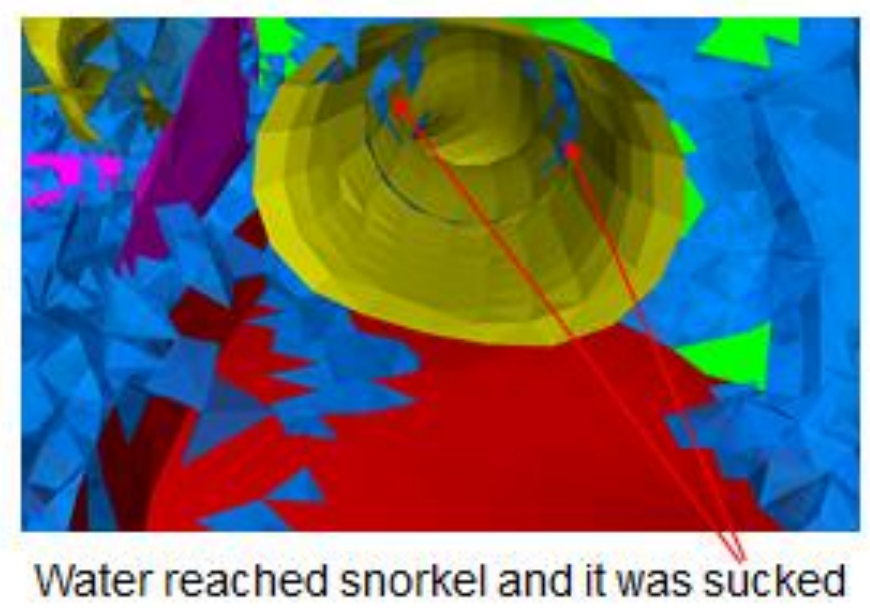

Figure 5. Snorkel inlet representation on the simulation at 4 seconds of the simulation

The physical test also presented water at the air filter after crossing the water height of $300 \mathrm{~mm}$ at $20 \mathrm{kph}$, which is the same simulation condition. Figure 6 shows the image of the air filter after the test and water inside the part.

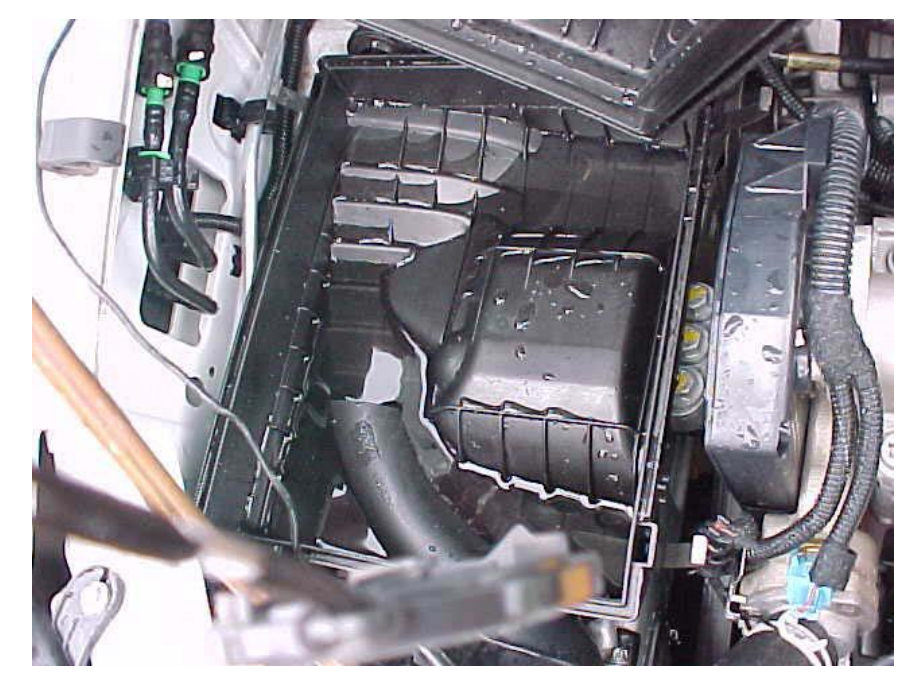

Figure 6. Air Filter with water after the vehicle crosses $300 \mathrm{~mm}$ water height at $20 \mathrm{kph}$

Comparing figure 5 and figure 6 it is possible to conclude that for both cases, water reached AIS system and the simulation methodology proposed in this paper was capable of predicting the same behavior of the physical test condition. 
The methodology can be applied to evaluate parts performance, AIS and water ingestion possibility by the engine at early stages of the project, which could lead to better designs for parts and systems before any physical test is performed

\section{Complementary Conclusions}

As a complementary conclusion for this study, the pressure due to water acting on certain parts of the vehicle was evaluated in order to identify the pressure contour and magnitude over the car.

Considering the air and the water on the system, Figure 7 shows the pressure contour over the fascia of the vehicle at simulation time of 4 seconds, as a stage water flow.

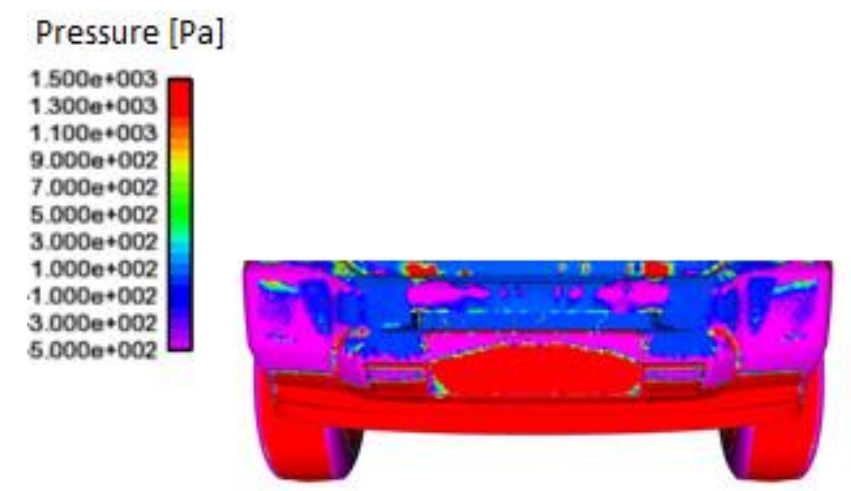

Figure 7. Pressure contour over the vehicle at simulation time of 4 seconds

This complementary result shown the pressure acting on the parts of the vehicle due to water splash can be used to evaluate fixations due to the flood condition. It can be used as one type of Fluid-Structure Interaction simulation, once CFD inputs are used on a structural CAE simulation.

Results for this case of pressure condition are only available virtually, once for the physical test, only the water ingestion was monitored.

As complementary works, a correlation for the pressure loads considering both physical and test would be a great work, once this correlation may lead to evaluate structural performance of the part submitted to the water pressure load. And for this case, it could help on improve both vehicle's development time and cost.

Another complementary work would be a use of a coupled CFD-CAE solver or Direct FSI in order to evaluate parts deformation together with the CFD multiphase simulation in order to improve more the confidence level of the simulation.

\section{REFERENCES}

1. BUSCARIOLO, F.F.; KARBON, K.J., Comparative CFD Analysis Between Rotating and Static Cases of Different Wheels Opening Designs over a Performance Sedan, SAE Paper, No 2011-36-0271, Society of Automotive Engineers, 2011. 
2. FLUENT 14.0., User's Guide, Fluent Inc., 2011.

3. HUCHO, W. H., Aerodynamics of Road Vehicles, From Fluid Mechanics of Vehicle Engineering, 4th Edition, SAE International, 1998.

4. Hypermesh 11.0 User's manual Altair Computing, Inc., 2011.

5. KATZ, J., Race Car Aerodynamics: Designing for Speed, Bentley Publishers, 1995.

6. KELlY, K. B.; PROVENCHER, L. G.; SCHENKEL, F. K., The General Motors Engineering Staff Aerodynamics Laboratory - A full Scale Automotive Wind Tunnel, SAE Paper, N 820371, Society of Automotive Engineers, 1982.

7. WHITE, F., Fluid Mechanics - fouth edition, McGraw Hill, 1999

8. WIEDEMANN, J., The Influence of Ground Simulation and Wheel Rotation on Aerodynamic Drag Optimization - Potential for Reducing Fuel Consumption, SAE Paper, No 960672, Society of Automotive Engineers, 1996. 